\title{
Band electron spectrum and optical properties of KDP-crystals under the external hydrostatic pressure
}

\author{
R.Ya.Stetsiv \\ Institute for Condensed Matter Physics \\ of the National Academy of Sciences of Ukraine, \\ 1 Svientsitskii Str., 290011 Lviv, Ukraine
}

Received March 6,1998

\begin{abstract}
The electron spectrum of the KDP-type crystals has been investigated as a function of the external hydrostatic pressure using the tight-binding approximation. The joint density of electron states, real and imaginary parts of the dielectric permeability, refraction indices, the gyration coefficient, absorption and reflection coefficients for different polarizations of light are determined. Their pressure and frequency dependencies are investigated. The results are discussed by comparing them with the experimental data on piezooptic coefficients. The anomalous behaviour of the optical constants at the pressure $p \simeq 17 \mathrm{kbar}$ is due to the transformation of the hydrogen bond potential from a double minimum one to a single minimum one, where the proton is localised at the midpoint of the hydrogen bond.
\end{abstract}

Key words: electron spectrum, hydrostatic pressure, optical properties

PACS: $78.20 .-y$

\section{Introduction}

The physical properties of ferroelectric systems with hydrogen bonds are mainly functions of the bond length and the character of proton motions. Above $T_{\mathrm{c}}$, the protons move in a symmetric double well potential along the $O-H-O$ bonds. Below the ferroelectric transition temperature $T_{\mathrm{c}}$, an ordering of protons in one of these two minima takes place. The most important effect, connected with the decreasing of the bond length, is the decreasing and ultimate vanishing of the transition temperature $T_{\mathrm{c}}[1]$. So, it is interesting to investigate the physical properties of such systems with a change of the structural parameters of hydrogen bonds, which gives us a possibility to examine the mechanism of the ferroelectric phase transition. One can change the structure of hydrogen bonds by external pressure, deuteration or other isomorphic changes, for example, substitution of 
$\mathrm{KH}_{2} \mathrm{PO}_{4}$ by $\mathrm{RbH}_{2} \mathrm{PO}_{4}, \mathrm{KH}_{2} \mathrm{AsO}_{4}$, etc. Application of pressure is the simplest of them, because the system itself does not change. It is known (see $[2,3])$ that both $\mathrm{KDP}$ and DKDP show a large negative pressure dependence of $T_{\mathrm{c}}$, and $T_{\mathrm{c}}$ of KDP falls to $0 \mathrm{~K}$ at $17 \mathrm{kbar}$. According to Nelmes and co-authors [4-9], above $17 \mathrm{kbar}$, at $0 \mathrm{~K}$, the along-bond (zero-point) thermal motion of the $\mathrm{H}$ atom about each site (i. e. the two minima of the potential well with the distance $\delta$ between them), $U^{22}(\mathrm{H})$ is larger than $(\delta / 2)^{2}$. Strictly, $U^{22}(\mathrm{H})$ is a mean-square amplitude along the $y$-axis; but this axis is very close to the line joining the two $\mathrm{H}$ sites. There then seems to be a simple structural explanation for the loss of the ordered phase that does not necessarily entail tunnelling effects: above $17 \mathrm{kbar}$, the thermal amplitude of the $\mathrm{H}$ atom is always greater than $\delta / 2$, the distance from each of the sites to their midpoint, and it seems reasonable to expect that the $\mathrm{H}$ atom cannot be localised on one site only. The experimental data of [4-9] describe the changes of the KDP structure with pressure in the pressure region 1 bar $\leqslant p \leqslant 17 \mathrm{kbar}$. In paper [12], similar investigations for the pressures 1 bar $\leqslant p \leqslant 54$ kbar were carried out, but the proton positions were not determined. Thus, the behaviour of the protons in the KDP structure at pressure $p>17 \mathrm{kbar}$ is not experimentally studied. According to [10,11], at further decrease of the bond length (for example, with the increase of pressure, for $p>17 \mathrm{kbar}$ ), the $\delta$ value falls to zero. This change may be either abrupt [11] or smooth but in the latter case $\delta$ rapidly nonlinearly falls to zero [10]. According to $[10,12]$, this change is expected in the pressure region $17 \mathrm{kbar} \leqslant p \leqslant 27 \mathrm{kbar}$. The value of pressure at which $\delta \rightarrow 0$ can be determined only experimentally. It is interesting to calculate the changes of optical functions which are induced by an abrupt change of $\delta \rightarrow 0$. We have taken $\delta \rightarrow 0$ at $p=17 \mathrm{kbar}$ in our calculations of optical constants. The results will not change qualitatively if we put $\delta \rightarrow 0$ at other pressure values of the described region up to $27 \mathrm{kbar}$, only the corresponding anomaly of optical functions will be shifted to higher values of pressure.

It was shown $[4-9,12]$ that the structural parameters such as the hydrogen bond length $(2 \mathrm{R})$, the distance between two proton-sites on a hydrogen bond $(\delta)$ and the rotation angle of $\mathrm{PO}_{4}$-groups around the $c$-axis $(\theta)$ vary linearly with pressure in a pressure region from 1 bar to $17 \mathrm{kbar}: \Delta \theta / \Delta p=5.8 \cdot 10^{-2}$ degree $/ \mathrm{kbar}$, $\Delta(2 R) / \Delta p=-1.8 \cdot 10^{-3} \AA / \mathrm{kbar}, \Delta(\delta) / \Delta p=-3.2 \cdot 10^{-3} \AA / \mathrm{kbar}$. For the lattice parameters $a$ and $c$ we have: $\Delta c / \Delta p=-7.152 \cdot 10^{-3} \AA / \mathrm{kbar},(\Delta a / \Delta p) \approx-7.46$. $10^{-3} \AA /$ kbar. Some of these parameters $(2 R, \theta, a, c)$ continue to vary linearly at pressures up to $27 \mathrm{kbar}[12]$.

There are anomalous changes of some structural parameters at $\mathrm{p}=27 \mathrm{kbar}$. The bond length decreases almost linearly with the increase of pressure, but at pressure values larger than $27 \mathrm{kbar}$ it becomes much longer. The rotation angle $\theta$ increases abruptly at $27 \mathrm{kbar}$, too. These changes are probably connected with the softening of $\mathrm{PO}_{4}$ groups, which was observed experimentally at pressure values $p>27$ kbar [12].

In this work we have investigated an electron band structure of the KDPtype crystals as a function of the external hydrostatic pressure. We have used the 
experimental data on the structural changes induced by pressure. The joint density of electron states, real and imaginary parts of the dielectric permeability, refraction indices, absorption and reflection coefficients for different polarizations of light are determined. Their pressure and frequency dependencies are investigated.

There are no other band structure calculations in the literature to compare with, and the dielectric response has not been studied by other authors at frequencies which correspond to the energies of electron transitions. Up till now, calculations of separated groups $\mathrm{H}_{n} \mathrm{PO}_{4}(\mathrm{n}=0,1, \ldots, 4)$ electronic spectra have been presented $[13,14,15]$. The obtained $[14,15]$ spectra were used to calculate the contribution of $\mathrm{H}_{n} \mathrm{PO}_{4}$ groups to the refractive index and the gyration effect.

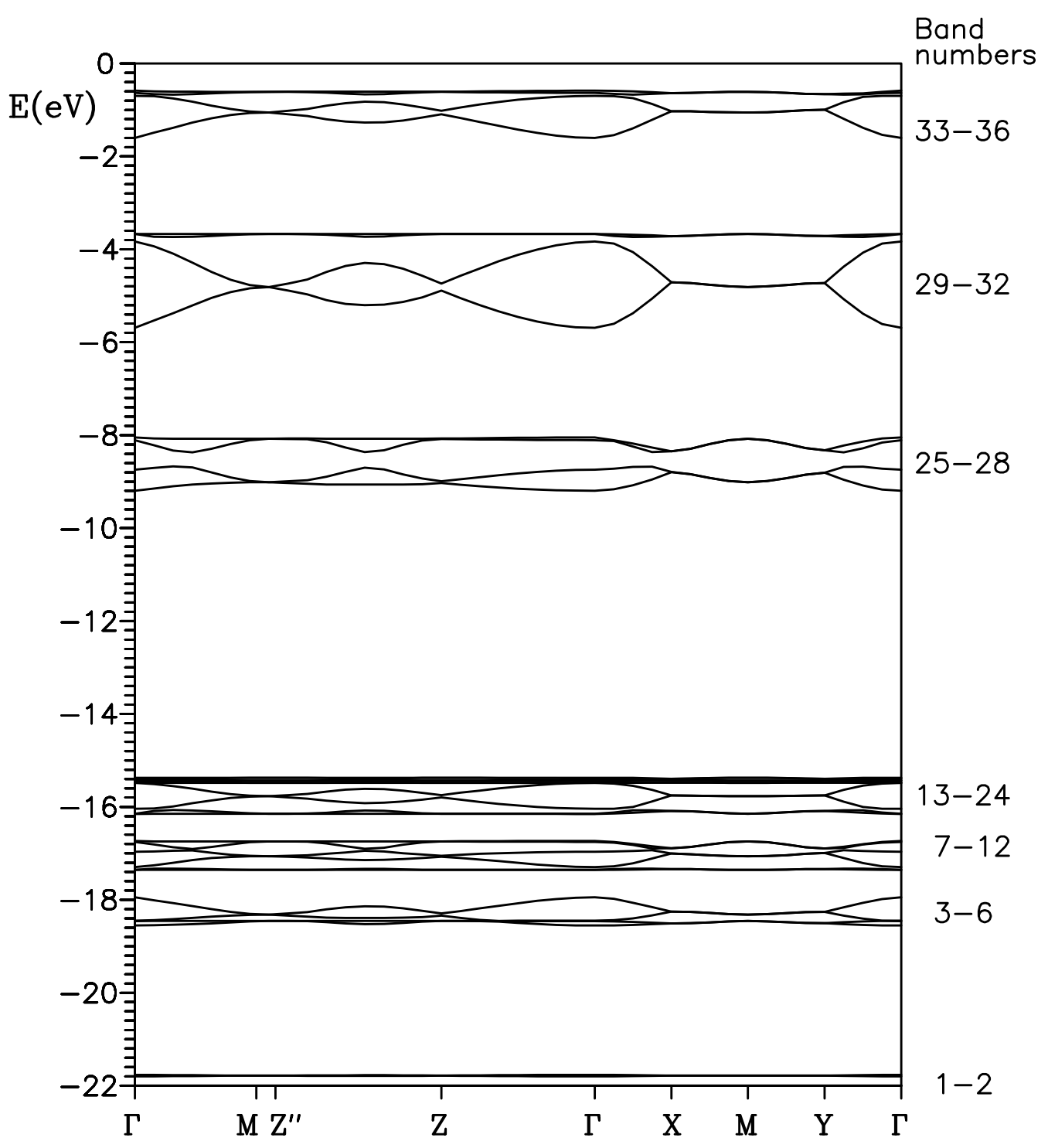

Figure 1. Electron band spectrum for a $\mathrm{KH}_{2} \mathrm{PO}_{4}$ crystal. 
Table 1. The values of the electron energies (in $\mathrm{eV}$ ) at $\Gamma, \mathrm{M}$ and $\mathrm{X}$ points of the Brillouin zone for the upper occupied band (number 24) and a set of the low-lying empty bands (numbers 25-31) at various values of pressure.

\begin{tabular}{|c|c|c|c|c|c|c|c|c|}
\hline \multirow[b]{2}{*}{$p$ (kbar) } & \multicolumn{8}{|c|}{ band number } \\
\hline & 31 & 30 & 29 & 28 & 27 & 26 & 25 & 24 \\
\hline & \multicolumn{8}{|c|}{$\overline{\Gamma-p o i n t}$} \\
\hline 0.001 & -3.6716 & -3.8348 & -5.6901 & -8.0494 & -8.1058 & -8.7409 & -9.1949 & -15.3636 \\
\hline $17.0-0.0$ & -3.6716 & -3.8169 & -5.7026 & -8.0140 & -8.0651 & -8.6080 & -9.0769 & -15.3636 \\
\hline \multirow[t]{2}{*}{$17.0+0.0$} & -3.6716 & -3.7744 & -5.7351 & -8.0439 & -8.0439 & -8.5816 & -9.0695 & -15.3636 \\
\hline & \multicolumn{8}{|c|}{ M-point } \\
\hline 0.001 & -3.6716 & -4.8128 & -4.8128 & -8.0777 & -8.0777 & -9.0109 & -9.0109 & -15.3636 \\
\hline $17.0-0.0$ & -3.6716 & -4.8062 & -4.8062 & -8.0396 & -8.0396 & -8.8892 & -8.8892 & -15.3636 \\
\hline \multirow[t]{2}{*}{$17.0+0.0$} & -3.6716 & -4.8078 & -4.8078 & -8.0439 & -8.0439 & -8.8759 & -8.8759 & -15.3636 \\
\hline & \multicolumn{8}{|c|}{$\mathrm{X}$-point } \\
\hline 0.001 & -3.7167 & -4.7119 & -4.7120 & -8.3453 & -8.3453 & -8.7931 & -8.7932 & -15.3732 \\
\hline $17.0-0.0$ & -3.7193 & -4.7240 & -4.7243 & -8.2817 & -8.2817 & -8.6884 & -8.6885 & -15.3732 \\
\hline $17.0+0.0$ & -3.7223 & -4.7267 & -4.7270 & -8.3057 & -8.3058 & -8.6543 & -8.6545 & -15.3732 \\
\hline
\end{tabular}

\section{Band electron spectrum of the $\mathrm{KH}_{2} \mathrm{PO}_{4}$ crystal}

The electron energy spectrum of the KDP-type crystals is calculated using the tight-binding approximation. The electron wave functions are presented as linear combinations of the Bloch functions which were constructed from the $3 \mathrm{~s}$ and $3 \mathrm{p}$ orbitals of phosphorus, $2 \mathrm{p}$ oxygen and 1s hydrogen orbitals:

$$
\psi_{\vec{q}}(\vec{r})=\frac{1}{\sqrt{N}} \sum_{n k s} \mathrm{e}^{\mathrm{i} \vec{q} \vec{R}_{n k}} \cdot C_{k s}(\vec{q}) \cdot \varphi_{n k s}\left(\vec{r}-\vec{R}_{n k}\right) .
$$

Here $n$ is a unit cell number, $k$ denotes an ion type and $s$ is an index of electron states. The atomic orbitals $\varphi_{n k s}$ were chosen in the form of Slater functions. A set of linear equations for the expansion coefficients $C_{k s}(\vec{q})$ is obtained from the solution of the Schrödinger equation with wave functions (2.1) using an extended Huckel method (see [16]):

$$
\sum_{k s}\left[\tilde{H}_{k^{\prime} s^{\prime}, k s}(\vec{q})-E(\vec{q}) \cdot \tilde{S}_{k^{\prime} s^{\prime}, k s}(\vec{q})\right] \cdot C_{k s}(\vec{q})=0
$$

Secular equations (2.3) provide a band energy spectrum

$$
\left|\tilde{H}_{k^{\prime} s^{\prime}, k s}(\vec{q})-E(\vec{q}) \cdot \tilde{S}_{k^{\prime} s^{\prime}, k s}(\vec{q})\right|=0,
$$

where

$$
\tilde{S}_{k^{\prime} s^{\prime}, k s}(\vec{q})=\sum_{n} S_{n^{\prime} k^{\prime} s^{\prime}, n k s} \cdot \mathrm{e}^{\mathrm{i} \vec{q}\left(\vec{R}_{n k}-\vec{R}_{n^{\prime} k^{\prime}}\right)}
$$

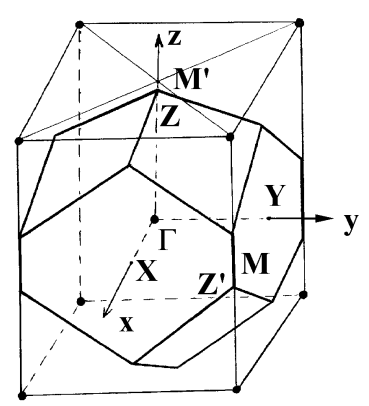

Figure 2. The first Brillouin zone for a $\mathrm{KH}_{2} \mathrm{PO}_{4}$ crystal. The line $\mathrm{ZM}^{\prime}$ is equivalent to the $\mathrm{Z}^{\prime} \mathrm{M}$ line. 


$$
\begin{gathered}
\tilde{H}_{k^{\prime} s^{\prime}, k s}(\vec{q})=\sum_{n} H_{n^{\prime} k^{\prime} s^{\prime}, n k s} \cdot \mathrm{e}^{\mathrm{i} \vec{q}\left(\vec{R}_{n k}-\vec{R}_{n^{\prime} k^{\prime}}\right)}, \\
S_{n^{\prime} k^{\prime} s^{\prime}, n k s}=\int \varphi_{n^{\prime} k^{\prime} s^{\prime}}\left(\vec{r}-\vec{R}_{n^{\prime} k^{\prime}}\right) \cdot \varphi_{n k s}\left(\vec{r}-\vec{R}_{n k}\right) \mathrm{d} V, \\
H_{n^{\prime} k^{\prime} s^{\prime}, n k s}=\int \varphi_{n^{\prime} k^{\prime} s^{\prime}}\left(\vec{r}-\vec{R}_{n^{\prime} k^{\prime}}\right) \cdot \hat{H} \varphi_{n k s}\left(\vec{r}-\vec{R}_{n k}\right) \mathrm{d} V,
\end{gathered}
$$

The summation in equations (2.4) and (2.5) was done over the nearest neighbours only. Secular equation (2.3) of the 36 th order with complex matrix elements was solved for the arbitrary wave vector $\vec{q}$. Here we take into account the structure changes induced by the external hydrostatic pressure. The dependence of the $R_{\mathrm{O}-\mathrm{H}}$

distance as well as the rotation angle of $\mathrm{PO}_{4}$-groups around the c-axis on pressure results in a pressure dependence of overlap integrals (2.6) and matrix elements of the Hamiltonian (2.7) and, thus, the band electron spectrum becomes a function of the external hydrostatic pressure. The calculations of the overlap integrals (2.6) and the matrix elements (2.7) are described in detail in our previous paper (see [16]). The results of the band structure calculation at the atmospheric pressure ( $p \approx 1$ bar) for $\vec{q}$ directed along the principal directions of the first Brillouin zone (BZ) are shown in figure 1. The dependence of the electron spectrum on pressure at $\Gamma, \mathrm{M}$ and $\mathrm{X}$-points in $\mathrm{BZ}$ is presented in table 1 . The first $\mathrm{BZ}$ for a $\mathrm{KH}_{2} \mathrm{PO}_{4}$ crystal is shown in figure 2. The state numbers are classified according to energy values. The bands from (1) up to (24) are filled in the ground state. The obtained energy gap is $6.17 \mathrm{eV}$, which is somewhat less than the experimental results: $7.0 \mathrm{eV}$ [17], $7.62 \mathrm{eV}$ [18].

The most essential changes under the influence of hydrostatic pressure are observed for the electron energy bands which are formed mostly by $1 s$-orbitals of hydrogen atoms (their numbers are 25-28). There are groups of bands which do not change with pressure (numbers 1,$2 ; 7,8 ; 13,14 ; 31,32$ ). We can include in these groups the upper occupied bands (numbers 17-24) the changes of which are smaller than $2 \cdot 10^{-3} \mathrm{eV}$, with the pressure change from 1 bar to $18 \mathrm{kbar}$. The dependence on the wave vector $\vec{q}$ for these bands is very weak. The widths of these bands are less than $2 \cdot 10^{-2} \mathrm{eV}$, except for numbers $13,14,31,32$ which are of the order $4 \cdot 10^{-2} \div 1 \cdot 10^{-1} \mathrm{eV}$. It should be noted that at $\mathrm{q}=0$ the energy values for the majority of these bands coincide with the energies of the corresponding levels $(2 \mathrm{~B}, 4 \mathrm{~A}, 4 \mathrm{~B}, 8 \mathrm{~B})$ of a separate group $\mathrm{H}_{2} \mathrm{PO}_{4}^{-}$with the point symmetry $C_{2}$, up to $1 \cdot 10^{-3} \mathrm{eV}[14]$.

\section{Optical properties}

The imaginary part of dielectric susceptibility was calculated from the following relation [19]:

$$
\varepsilon_{2}(\omega)=\frac{4 \pi^{2} e^{2}}{m^{2} \omega^{2}} \sum_{i, j} \int \frac{2 \mathrm{~d} \vec{q}}{(2 \pi)^{3}}\left|\vec{e} \vec{d}_{i j}(\vec{q})\right|^{2} \delta\left(E_{i}(\vec{q})-E_{j}(\vec{q})-\hbar \omega\right),
$$


Table 2. The calculated values of the piezooptic coefficients for a $\mathrm{KH}_{2} \mathrm{PO}_{4}$ crystal for various lengths of a light wave (in $10^{-13} \mathrm{~cm}^{2} /$ dyn).

\begin{tabular}{|c|c|c|c|c|c|c|}
\hline$\lambda(\mathrm{nm})$ & 400 & 500 & 600 & 800 & 1000 & 1200 \\
\hline $\mathrm{d} n_{o} / \mathrm{d} p$ & 14.4 & 14.3 & 14.0 & 14.0 & 14.0 & 14.0 \\
\hline $\mathrm{d} n_{e} / \mathrm{d} p$ & 15.0 & 15.0 & 15.0 & 15.0 & 15.0 & 15.0 \\
\hline $\mathrm{d} \varepsilon_{x x}^{-1} / \mathrm{d} \sigma$ & 7.39 & 7.69 & 7.71 & 7.90 & 7.97 & 8.02 \\
\hline $\mathrm{d} \varepsilon_{z z}^{-1} / \mathrm{d} \sigma$ & 7.50 & 7.89 & 8.10 & 8.32 & 8.40 & 8.45 \\
\hline
\end{tabular}

where

$$
\vec{e} \cdot \vec{d}_{i j}(\vec{q})=\left\langle\psi_{i \vec{q}}|\vec{e} \cdot \vec{p}| \psi_{j \vec{q}}\right\rangle=\vec{e} \cdot \int \psi_{i}^{*}(\vec{q}, \vec{r})(-\mathrm{i} \hbar \nabla) \cdot \psi_{j}(\vec{q}, \vec{r}) \mathrm{d} \vec{r} .
$$

The indices $j$ and $i$ denote the occupied and empty bands, respectively, and $\vec{e}$ is a unit vector of the polarization of light. Integration in equation (3.1) is performed over the first BZ. The real part of the dielectric susceptibility $\varepsilon_{1}$ for all frequencies is determined by the Kramers-Kronig dispersion relation:

$$
\varepsilon_{1}(\omega)=1+\frac{2}{\pi} P \int_{0}^{\infty} \omega^{\prime} \varepsilon_{2}\left(\omega^{\prime}\right) \frac{1}{\omega^{\prime 2}-\omega^{2}} \mathrm{~d} \omega^{\prime}
$$

Besides that, $\varepsilon_{1}$ within the transparency region was also calculated from the following relation [19]:

$$
\varepsilon_{1}(\omega)=1+\frac{8 \pi^{2} e^{2} \hbar^{2}}{m^{2}} \sum_{i, j} \int \frac{2 \mathrm{~d} \vec{q}}{(2 \pi)^{3}} \cdot \frac{\left|\vec{e} \vec{d}_{i j}(\vec{q})\right|^{2}}{\left[E_{i}(\vec{q})-E_{j}(\vec{q})\right]} \cdot \frac{1}{\left[E_{i}(\vec{q})-E_{j}(\vec{q})\right]^{2}-(\hbar \omega)^{2}} .
$$
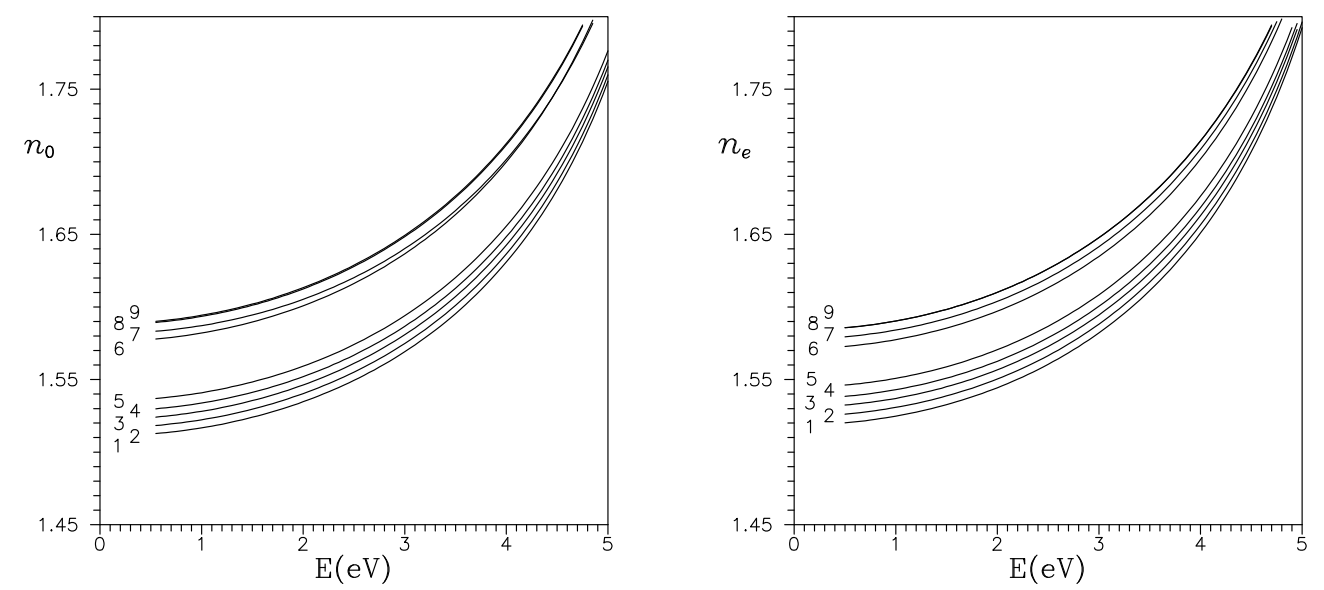

Figure 3. Spectral dependencies of the refraction indices $n_{o}$ and $n_{e}$ for $\mathrm{KH}_{2} \mathrm{PO}_{4}$ for various values of hydrostatic pressure: $1-1$ bar, $2-4 \mathrm{kbar}, 3-8 \mathrm{kbar}, 4-$ 12 kbar, 5 - (17.0 - 0.0) kbar, 6 - (17.0+0.0) kbar, 7 - 22 kbar, 8 - (27.0 - 0.0) kbar, $9-(27.0+0.0)$ kbar. 

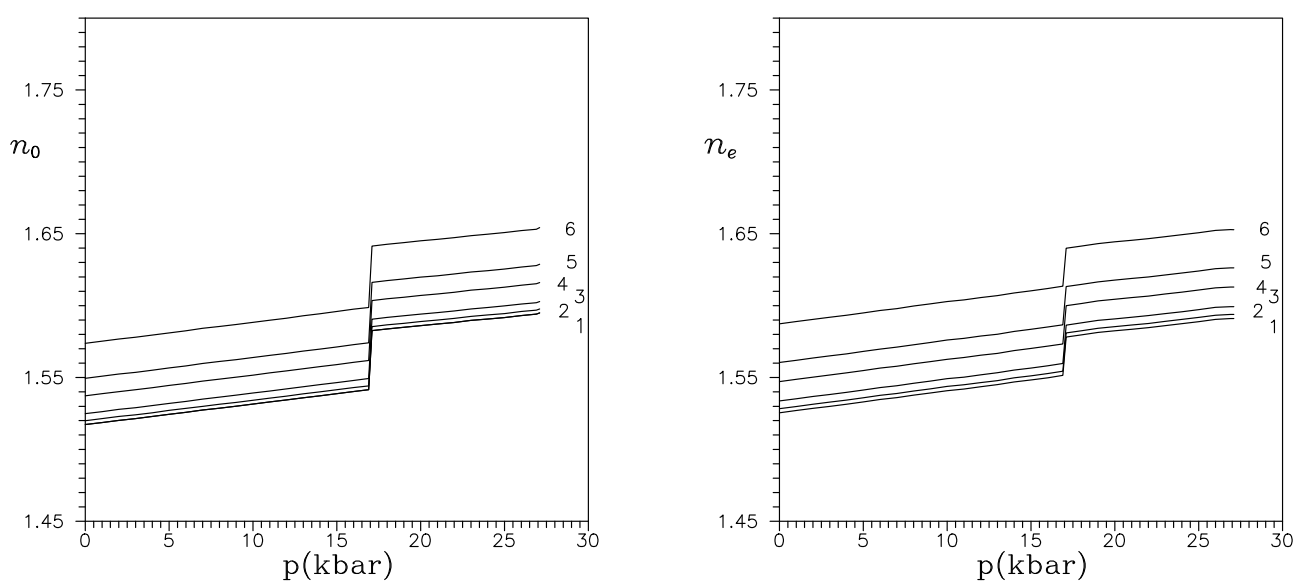

Figure 4. The pressure dependence of the refraction indices $n_{o}$ and $n_{e}$ for $\mathrm{KH}_{2} \mathrm{PO}_{4}$ for various lengths of a light wave: $1-1200 \mathrm{~nm}, 2-1000 \mathrm{~nm}, 3-$ $800 \mathrm{~nm}, 4-600 \mathrm{~nm}, 5-500 \mathrm{~nm}, 6-400 \mathrm{~nm}$.

The optical activity of crystals (i.e. gyrotropy) is a particular manifestation of spatial dispersion and is described by the linear term in the expansion of the dielectric permeability tensor $\varepsilon_{\alpha \beta}$ in powers of wave vector $\vec{q}[20]$ :

$$
\varepsilon_{\alpha \beta}(\omega, \vec{q})=\varepsilon_{\alpha \beta}^{(0)}(\omega)+\mathrm{i} \sum_{\gamma} A_{\alpha \beta, \gamma}^{(1)}(\omega) \cdot q_{\gamma}+\ldots
$$

We have calculated $g_{11}=\frac{\omega}{c} A_{y z, x}^{(1)}-$ a component of the gyration tensor, using the relation

$$
A_{\alpha \beta, \gamma}^{(1)}(\omega)=-8 \pi \hbar c \sum_{i, j} \int \frac{2 \mathrm{~d} \vec{q}}{(2 \pi)^{3}} \cdot \frac{\operatorname{Im}\left[d_{j i}^{\alpha}(\vec{q}) m_{i j}^{\beta \gamma}(\vec{q})+m_{j i}^{\alpha \gamma}(\vec{q}) d_{i j}^{\beta}(\vec{q})\right]}{\left[E_{i}(\vec{q})-E_{j}(\vec{q})\right]^{2}-(\hbar \omega)^{2}},
$$

where

$$
m_{i j}^{\beta \gamma}=\left\langle\psi_{i \vec{q}}|\hat{m}| \psi_{j \vec{q}}\right\rangle=\int \psi_{i}^{*}(\vec{q}, \vec{r})\left(r^{\gamma} p^{\beta}-r^{\beta} p^{\gamma}\right) \cdot \psi_{j}(\vec{q}, \vec{r}) \mathrm{d} \vec{r}
$$

The joint density of electron states is given by [19]:

$$
J_{i j}(\omega)=\int \frac{2 \mathrm{~d} \vec{q}}{(2 \pi)^{3}} \delta\left[E_{i}(\vec{q})-E_{j}(\vec{q})-\hbar \omega\right] .
$$

It provides the density of the pairs of states; one of them is occupied and the other is vacant, their energies differ by $\hbar \omega$.

The integrals in equations $(3.1),(3.4),(3.6),(3.8)$ were replaced by the sums over points $\vec{q}$ in the first BZ. We included 1836 points in the BZ, then the accuracy of the results is within $10 \%$. At each $\vec{q}$ point, the band spectrum $E(\vec{q})$ and matrix elements of the electric $e \hat{d}$ and magnetic $\frac{e}{2 m c} \hat{m}$ dipole electron momenta, defined by equations (3.2) and (3.7), respectively, were calculated. The values of $\varepsilon_{1}(\omega)$ obtained from equation (3.4) and from the Kramers-Kronig relation (3.3) differ by 
less than $1 \%$. For the calculation of $\varepsilon_{2}$ from equation (3.1) and in equation (3.8) we approximated the $\delta$-function in the following way:

$$
\delta\left[E_{i}(\vec{q})-E_{j}(\vec{q})-\hbar \omega_{0}\right]=\left\{\begin{array}{cc}
1 / \Delta, & \left|E_{i}-E_{j}-\hbar \omega_{0}\right| \leqslant \Delta / 2 \\
0, & \left|E_{i}-E_{j}-\hbar \omega_{0}\right|>\Delta / 2
\end{array},\right.
$$

where parameter $\Delta$ is taken equal to $0.05 \mathrm{eV}$. We calculated the values for refractive indices $n$, extinction coefficients $K$, absorption coefficients $\alpha$ and reflection $R$. The formulas presented below were used:

$$
\begin{array}{cc}
n=\sqrt{\frac{1}{2}\left(\sqrt{\varepsilon_{1}^{2}+\varepsilon_{2}^{2}}+\varepsilon_{1}\right)}, & K=\sqrt{\frac{1}{2}\left(\sqrt{\varepsilon_{1}^{2}+\varepsilon_{2}^{2}}-\varepsilon_{1}\right)}, \\
\alpha(\omega)=\frac{2 \omega}{c} \cdot K(\omega), & R=\frac{(n-1)^{2}+K^{2}}{(n+1)^{2}+K^{2}} .
\end{array}
$$

We have found that the refractive indices $n_{0}$ and $n_{e}$ change almost linearly with pressure in the pressure region 1 bar $\leqslant p<17$ kbar. The calculated pressure and frequency dependencies of the optical constants are shown in figures $3-8$. Their anomalous behaviour at the pressure values $p=17 \mathrm{kbar}$ and $p=27 \mathrm{kbar}$ are due to the changes of an electron spectrum and wave functions (see table 1) which are induced by the structure changes described above. The obtained changes of the optical constants at $p=27$ kbar are less essential than at $p=17 \mathrm{kbar}$. It was taken into account that for pressure values above $p \simeq 17$ kbar the hydrogen bond is transformed from two-minimum to one-minimum bond, when the proton is localised at the midpoint of the hydrogen bond. The correlation between the

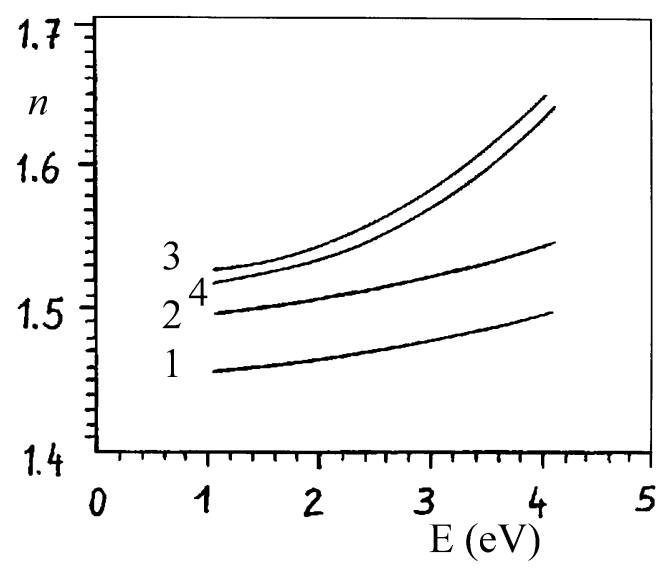

Figure 5. Spectral dependencies of the refraction indices for $\mathrm{KH}_{2} \mathrm{PO}_{4}$ at the atmospheric pressure ( $p=1$ bar). 1,2 - experimental data [22], 3,4 - calculated, $1,3-n_{e}, 2,4-n_{o}$. character of a proton distribution and the changes of $n_{0}, n_{e}$ and $g_{11}$ is essential, so it is interesting to study experimentally the behaviour of optical constants with the pressure change in order to investigate their possible anomalies at high pressures $(p \geqslant 17 \mathrm{kbar})$. Such investigations will give us a possibility to learn the behaviour of protons at high pressure and, thus, to examine the nature of the ferroelectric phase transition from a new point of view. The obtained dispersion of the refractive indices is stronger than the one observed experimentally (see figure 5). One can predict such results because we have obtained from the calculations the energy gap somewhat less than the experimental 
data (see above). It may be caused by the single-electron approximation used for band structure calculations. Besides, we did not use any fitting parameters in the calculation of the electron spectrum and optical constants.

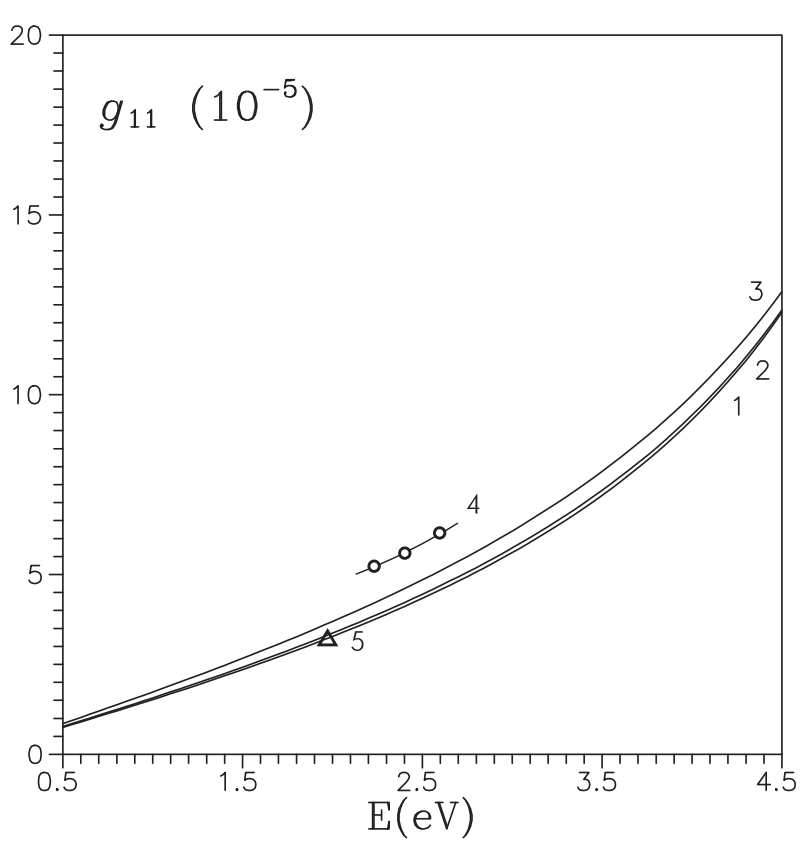

Figure 6. Spectral dependence of the $g_{11}$ component of the gyration tensor for $\mathrm{KH}_{2} \mathrm{PO}_{4}$ for various values of hydrostatic pressure: $1-1$ bar, $2-(17.0-0.0)$ kbar, $3-(17.0+0.0)$ kbar, 4 - 1 bar, 5 - 1 bar; 1,2,3 - calculated, 4,5 - experimental data [24], [25].

The main features of the obtained absorption and reflection spectra at the atmospheric pressure ( $p \approx 1$ bar) are in agreement with the experimental data $[13$, 17] (see figure 8). A wide absorption band at energies $8.5-10.0 \mathrm{eV}$ corresponds to the $\mathbf{A}$ band in the experiment, while the absorption bands at $10.5-11.2 \mathrm{eV}$ and $11.8-$ $12.7 \mathrm{eV}$ correspond to the $\mathbf{B}$ and C bands, respectively. A huge absorption band at $14.0-15.8 \mathrm{eV}$ coincides with the $\mathbf{D}$ band obtained experimentally. It should be noted that we have obtained the absorption bands in the energy region $6-8 \mathrm{eV}$, which are not observed experimentally.

Let us consider in more detail the dependence of the absorption spectra on hydrostatic pressure. An increase of the main maxima of the absorption and reflection spectra with pressure takes place.

It is characteristic especially of the $A$ maximum which lies in the frequency region $\hbar \omega \sim 9-10 \mathrm{eV}$. That effect is more essential for the light polarized perpendicularly to the $c$-axis. The dependence of the optical functions which describe the absorption and reflection of the light polarized parallelly to the $c$-axis (functions $K_{3}, \varepsilon_{2}^{z z}$ etc) on pressure is less essential. The anomalous increase of functions $K_{1}, \varepsilon_{2}^{x x}$ at pressure passing the value $p \simeq 17 \mathrm{kbar}$ is larger than the increase of these functions when the pressure is changed from 1 bar to $17 \mathrm{kbar}$ (see figure 7 ).

It is possible to determine piezooptic coefficients using the obtained pressure dependencies of the coefficients $n_{0}$ and $n_{e}$. In order to describe the change of the dielectric susceptibility $\varepsilon_{\alpha, \beta}$ with the pressure change we shall utilize the following relation:

$$
\varepsilon_{\alpha \beta}^{-1}(\omega \sigma)=\varepsilon_{\alpha \beta}^{(0)-1}(\omega)+\sum_{k e} \pi_{i j k e} \sigma_{k e}
$$

where $\pi_{i j k e}$ are piezooptic coefficients, $\sigma_{k e}$ is a tensor of mechanical strain. 

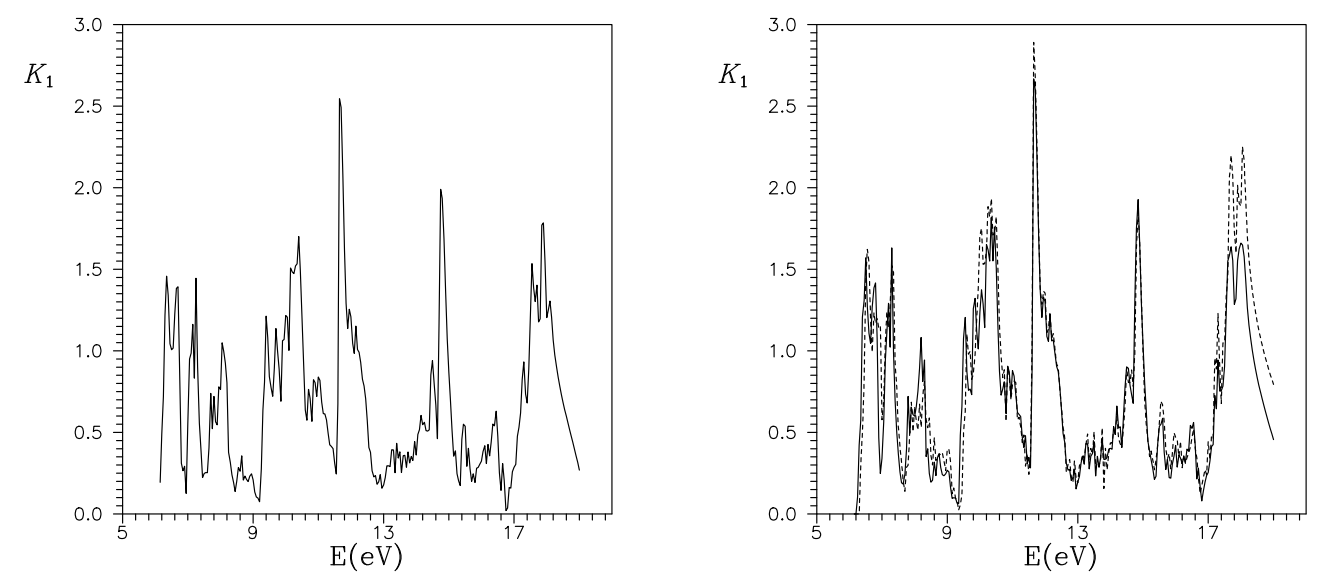

Figure 7. Spectral dependence of the extinction coefficient $K_{1}$ for the light polarized perpendicularly to the $c$-axis, for $\mathrm{KH}_{2} \mathrm{PO}_{4}$ at various values of hydrostatic pressure: (a) - 1 bar, (b) - (17.0 - 0.0) kbar - solid line, $(17.0+0.0)$ kbar dashed line.

In the case of a hydrostatic pressure we have:

$$
\frac{\mathrm{d}\left(\varepsilon_{x x}^{-1}\right)}{\mathrm{d} \sigma}=\pi_{11}+\pi_{12}+\pi_{13} \quad \frac{\mathrm{d}\left(\varepsilon_{z z}^{-1}\right)}{\mathrm{d} \sigma}=\pi_{31}+\pi_{32}+\pi_{33}
$$

We have found that the piezooptic coefficients $\mathrm{d}\left(\varepsilon_{x x}^{-1}\right) / \mathrm{d} \sigma$ and $\mathrm{d}\left(\varepsilon_{z z}^{-1}\right) / \mathrm{d} \sigma$ do not depend on pressure. Their changes with the change of pressure from 1 bar to $17 \mathrm{kbar}$ at fixed frequency are less than $2.5 \%$. The calculated values of the piezooptic coefficients are presented in table 2 . On the other hand, using the experimental data for $\pi_{i j}$ (for $\lambda=632.8$ ) nm $[21,22]$ we have: $\mathrm{d}\left(\varepsilon_{x x}^{-1}\right) / \mathrm{d} \sigma=$ $9.35 \cdot 10^{-13} \mathrm{~cm}^{2} / \mathrm{dyn} ; \mathrm{d}\left(\varepsilon_{z z}^{-1}\right) / \mathrm{d} \sigma=$ $7.64 \cdot 10^{-13} \mathrm{~cm}^{2} /$ dyn. So, the obtained piezooptic coefficients are in agreement with the experimental data. The $g_{11}$ component of the gyration tensor practically does not change with pressure, except for the anomalies at $\mathrm{p}=17$ kbar, when $g_{11}$ abruptly increases

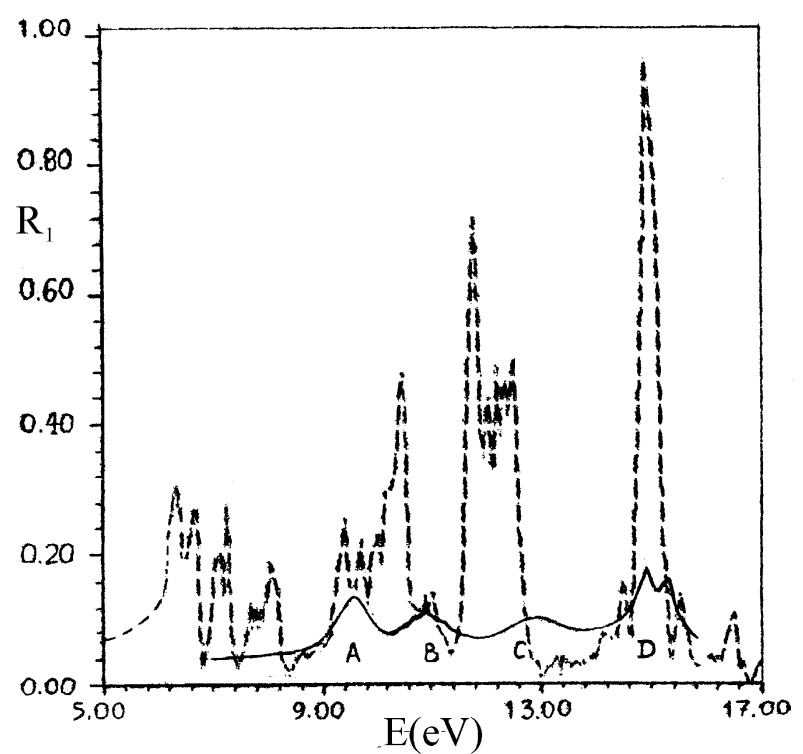

Figure 8. Spectral dependence of the reflection coefficient $R_{1}$ for $\mathrm{KH}_{2} \mathrm{PO}_{4}$ at the atmospheric pressure, solid line - experimental data [13], dashed line - calculated. approximately by $10 \%$. In the case of ionic contributions to the optical properties of a $\mathrm{KH}_{2} \mathrm{PO}_{4}$ crystal we also obtained earlier a weak increase of the $g_{11}$ component of the gyration tensor with the pressure change from 1 bar to 17 kbar [23]. 


\section{Acknowledgements}

It is my pleasure to thank Prof. I.Stasyuk for his steady attention to my research and many useful discussions. I am also thankful to Dr. A.Shvaika for his interest in the present work and for reading the manuscript.

The work has been supported in part by the Foundation for Fundamental Investigations of the Ukrainian Ministry in the Affairs of Science and Technology, Project No. 2.4/171.

\section{References}

1. McMahon M.C., Piltz R.O., Nelmes R.J. Neutron-diffraction studies of the relationship between $T_{\mathrm{c}}$ and H-bond dimensions in H-ordering transition. // Ferroelectrics, 1990, vol. 108, p. 277-282.

2. Samara G.A. The hydrogen bond in ferroelectricity and the role of high pressure research. // Ferroelectrics, 1978, vol. 20, p. 87-96.

3. Samara G.A. Pressure dependence of the static dielectric properties of $\mathrm{K}\left(\mathrm{H}_{1-x} \mathrm{D}_{x}\right)_{2} \mathrm{PO}_{4}$ and $\mathrm{RbH}_{2} \mathrm{PO}_{4}$. // Ferroelectrics, 1979, vol. 22, p. 925-936.

4. Nelmes R.J., Tibbals J.E. Structural studies of $\mathrm{KH}_{2} \mathrm{PO}_{4}$ and isomorphs as a function of temperature and pressure. // Ferroelectrics, 1981, vol. 39, p. 1041-1044.

5. Tibbals J.E., Nelmes R.J., Mc Inture G.J. The crystal structure of tetragonal $\mathrm{KH}_{2} \mathrm{PO}_{4}$ and $\mathrm{KDX}_{2} \mathrm{PO}_{4}$ as a function of temperature and pressure. // J. Phys. C., 1982, vol. 15, p. $37-58$.

6. Nelmes R.J.. Meyer G.M., Tibbals J.E. The crystal structure of tetragonal $\mathrm{KH}_{2} \mathrm{PO}_{4}$ and $\mathrm{KDX}_{2} \mathrm{PO}_{4}$ as a function of temperature. // J. Phys. C., 1982, vol. 15, p. 59-75.

7. Tibbals J.E., Nelmes R.J. The p-T dependence of the crystal structure of KDP and DKDP above $T_{\mathrm{c}}$. // J. Phys. C, 1982, vol. 15, p. L848-L853.

8. Nelmes R.J. Structural studies of KDP and the KDP-type transition by neutron and X-ray diffraction: 1970-1985. // Ferroelectrics, 1987, vol. 71, p. 87-123.

9. Nelmez R.J., Tun Z., Kuhs W.F. A compilation of accurate structural parameters for KDP and DKDP, and users guide to their crystal structures. // Ferroelectrics, 1987, vol. 71 , p. $125-141$.

10. Matsushita E., Matsubara T. Note on isotope effect in hydrogen bonded crystals. // Progr. of Theor. Physics, 1982, vol. 67, No. 1, p. 1-19.

11. Noda Y., Kasatani H., Watanabe Y., Terauchi H. Temperature dependence of hydrogen bond nature in $\mathrm{K}_{3} \mathrm{H}\left(\mathrm{SO}_{4}\right)_{2}$. // J. Phys. Soc. Jap., 1992, vol. 61, No. 3, p. 905-915.

12. Endo Sh., Chino T., Tsuboi Sh., Koto K. Pressure-induced transition of the hydrogen bond in the ferroelectric compounds $\mathrm{KH}_{2} \mathrm{PO}_{4}$ and $\mathrm{KDX}_{2} \mathrm{PO}_{4}$. // Nature, 1989, vol. 340 , p. $452-455$.

13. Saito S., Wada K., Onaka R. Vacuum ultraviolet reflection spectra of KDP and ADP. // J. Phys. Soc. Japan, 1974, vol. 37, No. 3, p. 711-715.

14. Stetsiv R.Ya., Yurechko R.Ya. Electron spectrum of ionic groups and the contribution of radiation defects into the optical activity of KDP-type crystals. Preprint of Inst. Teor. Phys. Natl. Acad. Sci. of Ukraine, ITF-88-52P, Kiev, 1988, 15 p. (in Russian).

15. Stasyuk I.V., Stetsiv R.Ya. Electron states and optical effects in KDP-type crystals with hydrogen bonds. // Izv. Acad. Nauk SSSR, Ser. fiz., 1991, vol. 55, p. 522. 
16. Stasyuk I.V., Stetsiv R.Ya. Band electron spectrum and optical constants of $\mathrm{KH}_{2} \mathrm{PO}_{4}$ crystal. Preprint of Institute for Condensed Matter Physics National Academy of Sciences of Ukraine, ICMP-92-30, Lviv, 1992, 29 p. (in Ukrainian).

17. Baldini G., Cottini M., Grilli E. Ultraviolet reflection and absorption of $\mathrm{KH}_{2} \mathrm{PO}_{4}$ and $\mathrm{NH}_{4} \mathrm{H}_{2} \mathrm{PO}_{4}$. // Solid State Communications, 1972, vol. 11, No. 9, p. 1257-1260.

18. Koralewski M., Szafranski M. On the energy gap in KDP-type crystals. // Acta Physica Polonica, 1984, vol. A66, No. 3, p. 229-235.

19. Bassani F., Pastori Parravicini G. Electron States and Optical Transitions in Solids. Pergamon Press, Oxford, New York, 1975.

20. Stasyuk I.V., Kotsur S.S. The microscopic theory of the gyration and electrogyration in dielectric crystals. // Phys. Stat. Sol.(b), 1983, vol. 117, No. 2, p. 557-568.

21. Narasimhamurty T.S. Photoelastic and Electro-optic Properties of Crystals. New York; London, 1981.

22. Blestanov A.A., Bondarenko V.S. et al. In: Ed. Shaskolskaya M.P. Acoustical Crystals. Handbook. Moscow, Nauka, 1982.

23. Stasyuk I.V., Stetsiv R.Ya, Golubets T.V. Influence of hydrostatic pressure on the electron spectrum of ionic groups and their contributions to optical properties of KDP-type crystals. // Ukrainian Journal of Physics, 1996, vol. 41, No. 10, p. 910915.

24. Kobayashi J. Optical activity and electrogyration of some improper ferroelectric crystals. // Memoirs School of Sci. and Eng., Waseda Univ., 1979, No 43, p. 1-26.

25. Vlokh O.G., Klepach N.I., Shopa Ya.I. Measurements of optical activity KDP-type crystals at phase transition // Kristallografiya, 1986, vol. 31, No. 1, p. 195-197.

\section{Зонний електронний спектр і оптичні властивості кристалів типу KDP при наявності зовнішнього гідростатичного тиску}

\section{Стеців Р.Я.}

Інститут фізики конденсованих систем НАН України, 290011 Львів, вул. Свєнціцького, 1

Отримано 6 березня 1998 р.

В наближенні сильно зв'язаних електронів досліджено залежність зонного електронного спектру кристалів типу KDP від зовнішнього гідростатичного тиску. Розраховані комбінована густина електронних станів, дійсна та уявна частини діелектричної проникності для різної поляризації світла, показники заломлення, коефіцієнт гірації, коефіцієнти поглинання та відбивання світла. Досліджена ї залежність від енергії світлової хвилі та від зовнішнього гідростатичного тиску. Результати порівнюються з експериментальними даними для п'єзооптичних коефіцієнтів. Отримані аномалії оптичних констант при тиску $p \simeq 17$ кбар зумовлені трансформацією потенціалу протона на зв'язку з двомінімумного в одномінімумний.

Ключові слова: електронний спектр, гідростатичний тиск, оптичні властивості

PACS: $78.20 .-y$ 